\title{
Effects of Juniper Essential Oil on the Activity of Autonomic Nervous System
}

\author{
Jong-Seong Park ${ }^{\dagger}$ \\ Department of Physiology, Chonnam National University Medical School, Gwangju 61469, Korea
}

This study was designed to clarify the effect of juniper essential oil on the autonomic nervous system. Blood pressure and heart rate variability (HRV) reflecting autonomic nervous system activity were measured. The systolic and diastolic blood pressure were decreased by inhalation of juniper essential oil. High frequency (HF) power level, an indicator of parasympathetic nervous system activity was increased in the stimulation of juniper essential oil. High frequency/low frequency $(\mathrm{HF} / \mathrm{LF})$ ratio, an indicator of sympathetic nervous system activity was decreased by the juniper essential oil. These results suggest that juniper essential oil has a modulatory effect on the autonomic nervous system activity.

Key Words: Juniper, Autonomic nervous system, Blood pressure

It is well known that olfactory stimulus has various effects on the emotion, behaviors and physiological functions (Liu et al., 2014). Olfactory stimulations are sensed by olfactory chemoreceptors in the nasal mucosa, which is connected to the primary olfactory regions in the central nervous system (CNS). Olfactory information in part are transmitted to the limbic system which controls the emotion and autonomic nervous system (McCabe and Rolls, 2007). A number of essential oils such as rose, lavender, bergamot, lemon, and sandalwood are currently in use as aromatherapy agents to relieve stress, anxiety, and depression (Mannucci et al., 2017). Inhaling the scent of lavender is reported to prevent stress, anxiety, and depression after childbirth, and inhalation of bergamot essential oil decreases behavior related depressive disorder (Kianpour et al., 2016; Saiyudthong and Mekseepralard, 2015).

Juniper is an evergreen coniferous shrub occurring in Northeastern Asia. It is reported to have various biologic effects (Rombolà et al., 2017). The berries of $J$. rigida have been used in Korean traditional medicine for the treatment of rheumatoid arthritis and the branches and leaves of $J$. rigida have been used for the treatment of nephritis and rheumatic arthritis (Zhao et al., 2016). Juniper essential oil is a safe and widely used material for aromatherapy (Barclay et al., 2006). The effect of juniper essential oil on the autonomic nervous system has not been reported. The change of autonomic nervous system activity after inhalation of essential oil can be detected by frequency domain analysis of heart rate variability (HRV) (TFESC, 1996). The aim of this study is to investigate the effects of juniper essential oil on the autonomic nervous function in terms of HRV.

Eighteen persons ranging from 22 to 29 years old (mean \pm S.D.: $25 \pm 2.0$ years old), volunteered to participate in the study. Persons with nasal and breathing disorder were excluded. All participants received explanations of the aims, procedures of the study and safety of the juniper essential oil.

* Received: April 25, 2017 / Revised: July 21, 2017 / Accepted: July 23, 2017

†Corresponding author: Jong-Seong Park. Department of Physiology, Chonnam National University Medical School, 42 Jebong-ro, Dong-gu, Gwangju 61469 , Korea.

Tel: +82-62-220-4264, Fax: +82-62-232-1242, e-mail: parkjs@jnu.ac.kr

(C) The Korean Society for Biomedical Laboratory Sciences. All rights reserved.

(c) This is an Open Access article distributed under the terms of the Creative Commons Attribution Non-Commercial License (http://creativecommons.org/licenses/by-nc/3.0/) which permits unrestricted non-commercial use, distribution, and reproduction in any medium, provided the original work is properly cited. 
Table 1. Effects of juniper essential oil on the blood pressure $(n=$ 18)

\begin{tabular}{lrrc}
\hline \hline & Control & Juniper & $P$ value \\
\hline SBP (mmHg) & $118 \pm 2$ & $107 \pm 3$ & $P<0.05$ \\
DBP (mmHg) & $75 \pm 1$ & $68 \pm 2$ & $P<0.05$ \\
HR (beats/min) & $74 \pm 2$ & $72 \pm 2$ & Nonspecific \\
\hline
\end{tabular}

Abbreviations: $\mathrm{SBP}=$ systolic blood pressure; $\mathrm{DBP}=$ diastolic blood pressure; $\mathrm{HR}=$ heart rate

The study was performed in accordance with the Declaration of Helsinki of the World Medical Association and Regulations of the Ethics Committee of Chonnam National University. All measurements were performed in a temperaturecontrolled $\left(25^{\circ} \mathrm{C}\right)$, quiet, comfortable room. Before measurements were taken, the participants relaxed comfortably for about $10 \mathrm{~min}$ in a seated position. Blood pressure measurements were done with electronic blood pressure detector (Omron HEM-7220, Omron Healthcare Co., Kyoto, Japan) Electrocardiogram (ECG) recording in the augmented unipolar limb leads was performed for the analysis of the HRV. HRV was analyzed by means of frequency domain analysis (SA-3000P, Medicore, Seoul, Korea). To apply juniper essential oil, a diffuser for aromatherapy was used (Aroma breeze NOVA T, ALTA Corporation, Nagoya, Japan). According to previous studies with essential oil, $10 \mu \mathrm{L}$ of juniper essential oil was pipetted onto a small cotton pad for a diffuse (Dunn et al., 1995). The speed of airflow was $1.2 \mathrm{~m} / \mathrm{min}$. The diffuser was placed near the participant's nostrils with $30-\mathrm{cm}$ long circular cylinder. The participants inhaled the essential oil diffused from the diffuser for $5 \mathrm{~min}$. HRV measurements were performed before and during the application of juniper oil. Blood pressure were performed before and just after the stimulation of juniper oil.

All data were expressed as mean \pm standard deviation. Student's $t$-test was used to compare the responses to the juniper essential oil. Values of $P<0.05$ were considered statistically significant. The systolic pressure was decreased by juniper essential oil from $118 \pm 2 \mathrm{mmHg}$ to $1107 \pm 3(P$ $<0.05)$. The diastolic pressure was also decreased from 75 $\pm 1 \mathrm{mmHg}$ to $68 \pm 2 \mathrm{mmHg}(P<0.05)$ by juniper essential oil. The heart rate also decreased from $74 \pm 2 /$ min to $72 \pm$
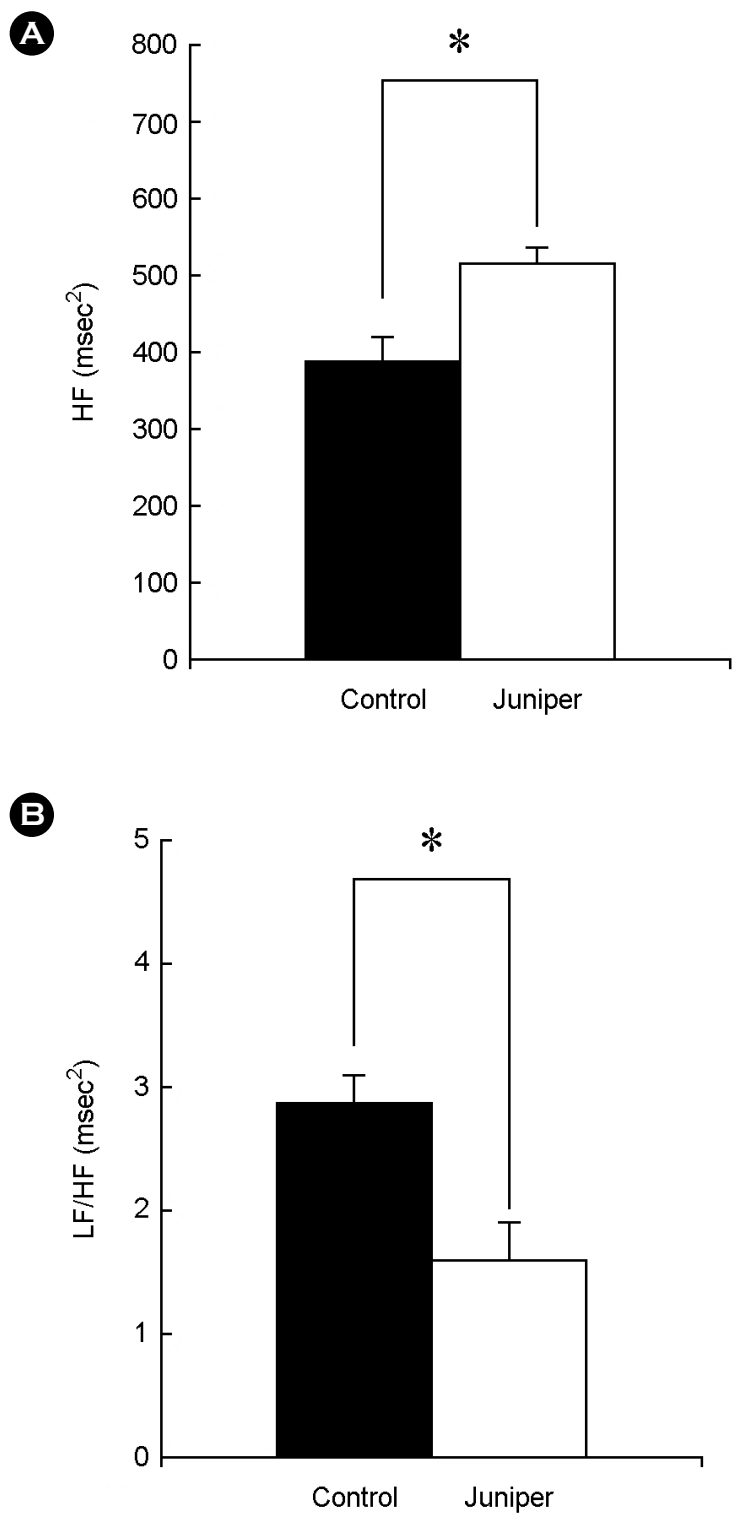

Fig. 1. Effects of juniper essential oil on high-frequency (HF) and low-frequency $(\mathrm{LF}) / \mathrm{HF}$ power level ratios of heart rate variability. Data are expressed as $\mathrm{n}=18 .{ }^{*} P<0.05$ by Student's $t$-test.

2/min without statistical significance (Table 1).

High frequency (HF) component was significantly higher $\left(509 \pm 19 \mathrm{msec}^{2}\right)$ with juniper application than that in the control condition $\left(385 \pm 30 \mathrm{msec}^{2}\right)(P<0.05)$. In contrast, changes in $\mathrm{LF} / \mathrm{HF}$ were significantly lower $(1.5 \pm 0.3)$ with juniper application in comparison to the control (2.8 \pm 0.2$)$ $(P<0.05)$. Low frequency (LF) component was significantly lower $\left(405 \pm 15 \mathrm{msec}^{2}\right)$ with juniper application than that in 
the control condition $\left(535 \pm 25 \mathrm{msec}^{2}\right)(P<0.05)$ (Fig. 1).

The heart is innervated by autonomic nervous system. Sympathetic nervous system accelerates the cardiac function, whereas parasympathetic nervous system decelerates the cardiac function. To evaluate the autonomic nervous system activity quantitatively, analyzing HRV in the frequency domain is known to be a valuable method (TFESC, 1996). By frequency domain analysis of oscillation in R-R intervals, $\mathrm{HF}(0.15 \sim 0.40 \mathrm{~Hz})$ reflects the activity of parasympathetic nervous system, and LF component $(0.04 \sim 0.15 \mathrm{~Hz})$ reflects both sympathetic and parasympathetic nervous activities (Peter et al., 2015). LF/HF is used as a relative marker of sympathetic nervous activity (Landolt et al., 2017).

There are a few reports on the effects of essential oils on the autonomic nervous system by measuring the HRV. Lavender inhalation elicits a significant increase in HF component and reduction in $\mathrm{LF} / \mathrm{HF}$ ratios, suggesting of increased parasympathetic nervous activity and reduced sympathetic activities (Saeki, 2000). Inhalation of petitgrain essential oil showed LF decrease and HF increase (Nagai et al., 2000).

It is well known that juniper essential oil has antimicrobial and antioxidant activity (Khoury et al., 2014). Juniper essential oil is reported to have ulceroprotective effects in rats and anti-acetylcholinesterase activities on amyloid Beta (1-42)-Induced oxidative stress in the rat hippocampus (Ben Ali et al., 2015). However, despite many studies on the biologic effects of juniper essential oil, its modulatory effects on the autonomic nervous system has not reported. In the present study, juniper increased HF with simultaneous reductions in the LF/HF ratios, which implies that juniper increases parasympathetic activities and decreases sympathetic activities. By gas chromatography-mass spectrometry, many components were identified from the juniper essential oil, including a-pinene, camphene, b-pinene, sabinene, myrcene, a-phellandrene, a-terpinene, y-terpinene caryophyllene, $\alpha$-caryophyllene, caryophyllene oxide, trans-nerolidol and germacrene (Ben Ali et al., 2015). It is not clear which component is related to the modulatory effects of juniper essential oil. But it could be postulated that elements of juniper essential oil absorbed by olfactory system into the CNS modulate the neuronal excitability, or control the neurotransmitter releasing process. (Vatanparast et al., 2017). Future studies are needed to investigate the precise mechanism of action of juniper essential oil.

\section{CONFLICT OF INTEREST}

The authors have no conflicts of interest to disclose.

\section{REFERENCES}

Barclay J, Vestey J, Lambert A, Balmer C. Reducing the symptoms of lymphoedema: is there a role for aromatherapy? European Journal of Oncology Nursing. 2006. 10: 140-149.

Ben Ali MJ, Guesmi F, Harrath AH, Alwasel S, Hedfi A, Ncib S, Landoulsi A, Aldahmash B, Ben-Attia M. Investigation of antiulcer and antioxidant activity of Juniperus phoenicea L. (1753) Essential Oil in an experimental rat model. Biological and Pharmaceutical Bulletin. 2015. 38: 1738-1746.

Dunn C, Sleep J, Collett D. Sensing an improvement: an experimental study to evaluate the use of aromatherapy, massage and periods of rest in an intensive care unit. Journal of Advanced Nursing. 1995. 1: 34-40.

Hellyer J, George Akingba A, Rhee KS, Tan AY, Lane KA, Shen C, Patel J, Fishbein MC, Chen PS. Autonomic nerve activity and blood pressure in ambulatory dogs. Heart Rhythm. 2014. 2: 307-313.

Khoury M1, El Beyrouthy M, Ouaini N, Iriti M, Eparvier V, Stien D. Chemical composition and antimicrobial activity of the essential oil of Juniperus excelsa M.Bieb. growing wild in Lebanon. Chemistry \& Biodiversity. 2014. 11: 825-830.

Kianpour M, Mansouri A, Mehrabi T, Asghari G. Effect of lavender scent inhalation on prevention of stress, anxiety and depression in the postpartum period. Iran Journal of Nursing and Midwifery Research. 2016. 21: 197-201.

Landolt K, O'Donnell E, Hazi A, Dragano N, Wright BJ. An experimental examination of the effort-reward imbalance model of occupational stress: Increased financial reward is related to reduced stress physiology. Biological Psychology. 2017. 125: 121-129.

Liu Y, Lieberwirth C, Jia X, Curtis JT, Meredith M, Wang ZX. Chemosensory cues affect amygdaloid neurogenesis and alter behaviors in the socially monogamous prairie vole. European Journal of Neuroscience. 2014. 39: 1632-1641.

McCabe C, Rolls ET. Umami: a delicious flavor formed by convergence of taste and olfactory pathways in the human brain. 
European Journal of Neuroscience. 2007. 25: 1855-1864.

Nagai M, Wada M, Usui N, Tanaka A, Hasebe Y. Pleasant odors attenuate the blood pressure increase during rhythmic handgrip in humans. Neuroscience Letter. 2000. 289: 227-229.

Peter R, Sood S, Dhawan A. Spectral parameters of HRV in yoga practitioners, athletes and sedentary males. Indian Journal of Physiology and Pharmacology. 2015. 59: 380-387.

Mannucci C, Navarra M, Calapai F, Squeri R, Gangemi S, Calapai G. Clinical pharmacology of citrus bergamia: a systematic review. Phytotherapy Research. 2017. 1: 27-39.

Saeki Y. The effect of foot-bath with or without the essential oil of lavender on the autonomic nervous system: a randomized trial. Complementary Therapies in Medicine. 2000. 8: 2-7.

Saiyudthong S, Mekseepralard C. Effect of inhaling bergamot oil on depression-related behaviors in chronic stressed rats. Journal of the Medical Association of Thailand. 2015. Suppl 9: S152-159.

Task Force of the European Society of Cardiology and the North
American Society of pacing and electrophysiology standards of measurement, physiological interpretation, and clinical use. Circulation Heart Rate Variability. 1996. 93: 1043-1065.

Vatanparast J, Bazleh S, Janahmadi M. The effects of linalool on the excitability of central neurons of snail Caucasotachea atrolabiata. Comparative Biochemistry and Physiology - Part C: Toxicology \& Pharmacology. 2017. 192: 33-39.

Zhao J, Liu T, Xu F, You S, Xu F, Li C, Gu Z. Anti-arthritic effects of total flavonoids from Juniperus sabina on complete Freund's adjuvant induced arthritis in rats. Pharmacognosy Magazine. 2016. 47: 178-183.

https://doi.org/10.15616/BSL.2017.23.3.286

Cite this article as: Park JS. Effects of Juniper Essential Oil on the Activity of Autonomic Nervous System. Biomedical Science Letters. 2017. 23: 286-289. 